\title{
An Improved Algorithm for Efficient Mining of Frequent Item Sets on Large Uncertain Databases
}

\author{
Bala Yesu Chilakalapudi \\ M.Tech Student \\ Gudlavalleru Engineering \\ College, Andhra Pradesh
}

\author{
Narayana Satyala \\ Associate Professor \\ Gudlavalleru Engineering \\ College, Andhra Pradesh
}

\author{
Satyanarayana Menda \\ Assistant Professor \\ Jayawant Engineering College \\ and Mgmt, Maharastra.
}

\begin{abstract}
The data handled in emerging applications like location based services, sensor monitoring systems, and data integration, are often inexact in nature. In this paper, the important problem of extracting frequent item sets from a large uncertain database, interpreted under the Possible World Semantics (PWS) is presented. This issue is technically challenging, since an uncertain database contains an exponential number of possible worlds. By observing that the mining process can be modeled as a Poisson binomial distribution, an algorithm was developed, which can efficiently and accurately discover frequent item sets in a large uncertain database. The important issue of maintaining the mining result for a database that is evolving (e.g., by inserting a tuple) can be presented. Specifically, the proposed mining algorithm can enable Probabilistic Frequent Item set (PFI) results to be refreshed. This reduces the need of re-executing the whole mining algorithm on the new database, which is often more expensive and unnecessary. The proposed algorithm can support incremental mining and provides the accurate results on mining the uncertain database. The extensive evaluation on real data set to validate the approach is performed.
\end{abstract}

\section{General Terms}

Frequent item sets, uncertain data set, incremental mining

\section{Keywords}

PFI, PWS, S-PMF, CDF.

\section{INTRODUCTION}

The data bases used in many important and novel applications are often uncertain. For example, the locations of users obtained through RFID and GPS systems are not precise due to measurement errors [22], [28]. As another example, data collected from sensors in habitat monitoring systems (e.g., temperature and humidity) are noisy [17]. Customer purchase behaviors, as captured in supermarket basket databases, contain statistical information for predicting what a customer will buy in the future [3], [6]. Integration and record linkage tools also associate confidence values to the output tuples according to the quality of matching [16]. In structured information extractors, confidence values are appended to rules for extracting patterns from unstructured data [31]. To meet the increasing application needs of handling a large amount of uncertain data, uncertain databases have been recently developed [10], [16], [19], [20], [27]. Performing the data mining under the possible world semantics(PWS) can be technically challenging. In fact, the mining of uncertain data has recently attracted research attention [3]. For example, in [23], efficient clustering algorithm was developed for uncertain objects; in [21] and [32], naive Bayes and decision tree classifiers designed for uncertain data were studied. Here ,the algorithm for finding frequent item sets (i.e., sets of attribute values that appear together frequently in tuples) for uncertain databases was developed. The proposed algorithm can be applied to two important uncertainty models: attribute uncertainty and tuple uncertainty, where every tuple is associated with a probability to indicate whether it exists [15], [16], [19], [27], [34]. The frequent item sets discovered from uncertain data are naturally probabilistic, in order to reflect the confidence placed on the mining results.

A probabilistic frequent item(PFI) is a set of attribute values that occurs frequently with a sufficiently high probability. A database induces a set of possible worlds, each giving a (different) support count for a given item set. Hence, the support of a frequent item set is described by a probability mass function (pmf). A simple way of finding PFIs is to mine frequent patterns from every possible world obtained by the possible world semantics (PWS), and then record the probabilities of the occurrences' of these patterns. This is impractical, due to the exponential number of possible worlds. To remedy this, some algorithm has been recently developed to successfully retrieve PFIs without instantiating all possible worlds [6], [30], [35]. These algorithm can verify whether an item set is a PFI in $\mathrm{O}\left(\mathrm{n}^{2}\right)$ time (where $\mathrm{n}$ is the number of tuples contained in the database). However, the experimental results reveal that they can require a long time to complete(e.g., with a $300 \mathrm{k}$ real data set, the dynamic programming algorithm in [6] needs 30.1 hours to find all PFIs). The support pmf of a PFI can be captured by a Poisson binomial distribution, for both attribute and tuple uncertain data. The proposed algorithm make use of this intuition to propose a method for approximating a PFI's pmf with a Poisson distribution, which can be efficiently and accurately estimated. This algorithm can verify a PFI in $\mathrm{O}\left(\mathrm{n}^{2}\right)$, and is thus more suitable for large databases. The algorithm can be used to mine the frequent item sets whose probabilities of being true frequent item sets are larger than some user defined threshold [6]. The algorithm only needs very less time to find all PFIs [33] when compared with the existing algorithm, which is four orders of magnitudes faster than the method used in [6].

\subsection{Mining evolving databases.}

The important problem of maintaining mining results for changing, or evolving, databases is prsented here. The type of evolving data that we address here is about the appending, or insertion of a batch of tuples to the database. Tuple insertion is common in the applications that we consider. For example, a GPS system may have to handle location values due to the registration of a new user; in an online marketplace application, information about new purchase transactions may 
be appended to the database for further analysis. Notice that these new tuples may induce changes to the mining result. A straightforward way of refreshing the mining results is to reevaluate the whole mining algorithm on the new database. This can be costly, however, when new tuples are appended to the database at different time instants. In fact, if the new database $\mathrm{D}$ is similar to its older version, $\mathrm{D}$, it is likely that most of the PFIs extracted from D remain valid for $\mathrm{D}^{+}$. Based on this intuition, The developed mining algorithm uses the PFIs of $\mathrm{D}$ to derive the PFIs of $\mathrm{D}^{+}$, instead of finding them from scratch. In this paper, The proposed mining algorithm for the method studied in [6], discovers the PFIs. The algorithm discovers PFIs, which can be extended to handle evolving data. As the experiments show, when the change of the database is small, running the mining algorithm on $\mathrm{D}^{+}$is much faster than finding PFIs on $\mathrm{D}^{+}$from scratch. In an experiment on a real data set, the mining algorithm addresses a fivefold performance improvement over its non counterpart.

To summarize, an algorithm was developed, which can reduce the amount of effort of scanning the database for mining threshold- based PFIs. The algorithm can support both attribute and tuple uncertainty models. The time complexity of the proposed approach is presented. Experiments on the real data set reveal that the proposed method significantly improves the performance of PFI discovery, with a high degree of accuracy. The rest of the paper is organized as follows: in Section 2, we review the related works. Section 3 defines the problems to be studied. Section 4 describes efficient and accurate methods for computing s- pmf. In Section 5, The working method of the algorithm for discovering threshold- based PFIs is presented. Section 8 reports the experimental results. The paper will conclude in Section 9.

\section{RELATED WORK}

Mining frequent item sets is an important problem in data mining, and is also the first step of deriving association rules [4]. Hence, many efficient item set mining algorithm(e.g., Apriori [4] and FP- growth [18]) have been proposed. While these algorithm work well for databases with precise values, it is not clear how they can be used to mine probabilistic data. Here an algorithm for extracting frequent item sets from uncertain databases was developed. Although the algorithm is developed based on the Apriori framework, they can be considered for supporting other algorithm (e.g., FP- growth) for handling uncertain data. For uncertain databases, Aggarwal et al. [2] and Chui et al. [14] developed efficient frequent pattern mining algorithm based on the expected support counts of the patterns. However, Bernecker et al. [6], Sun et al. [30], and Yiu et al. [35] found that the use of expected support may render important patterns missing. Hence, they proposed to compute the probability that a pattern is frequent, and introduced the notion of PFI. In [6], dynamic programming based solutions were developed to retrieve PFIs from attribute uncertain databases. However, their algorithm compute probabilities, and verify that an item set is a PFI in $\mathrm{O}\left(\mathrm{n}^{2}\right)$ time. the algorithm avoid the use of dynamic programming, and are able to verify a PFI much faster (in $\mathrm{O}(\mathrm{n})$ time). Zhang et al. [35] only considered the extraction of singletons (i.e., sets of single items), the solution discovers patterns with more than one item. Recently, Sun et al. [30] developed an threshold based PFI mining algorithm. However, it does not support attribute uncertain data considered in this paper other works on the retrieval of frequent patterns from imprecise data include: [9], which studied frequent patterns on noisy data; [24], which examined association rules on fuzzy sets; and [26], which proposed the notion of a "vague association rule." However, none of these solutions are developed on the uncertainty models studied here. For evolving databases. A few mining algorithm that work for data have been developed. For example, in [11], the Fast Update algorithm (FUP) was proposed to efficiently maintain frequent item sets, for a database to which new tuples are inserted. the mining framework is inspired by FUP. In [12], the FUP2 algorithm was developed to handle both addition and deletion of tuples. ZIGZAG [1] also examines the efficient maintenance of maximal frequent item sets for databases that are constantly changing. In [13], a data structure, called CATS Tree, was introduced to maintain frequent item sets in evolving databases. Another structure, called CanTree [25], arranges tree nodes in an order that is not affected by changes in item frequency. The data structure is used to support mining on a changing database. To the best knowledge, maintaining frequent item sets in evolving uncertain databases has not been examined before. The proposed algorithm can also support attribute and tuple uncertainty models.

\section{PROBLEM DEFINITION}

Let $\mathrm{V}$ be a set of items. The algorithm adopt the following variant [6]: a database $D$ contains $\mathrm{n}$ tuples, or transactions. Each transaction, $t_{j}$ is associated with a set of items taken from V. Each item $v \in \mathrm{V}$ exists in $t_{j}$ with an existential probability $\operatorname{Pr}\left(v \in t_{j}\right) \epsilon(0,1]$, which denotes the chance that $\mathrm{v}$ belongs to $t_{j}$. Under the Possible World Semantics, D generates a set of possible worlds. Each world consists of a subset of attributes from each transaction, occurs with probability Priz $\left(w_{i}\right)$.

The probabilities are one, and the number of possible worlds is exponentially large. the goal is to discover frequent patterns without expanding D into possible worlds. Each transaction $t_{j} \in D$ is associated with a set of items and an existential probability $\operatorname{Pr}\left(t_{j}\right) \in(0,1]$ which indicates that $t_{j}$ exists in $\mathrm{D}$ with probabilityPr( $\left(t_{j}\right)$. Again, the number of possible worlds for this model is exponentially large. The problem of mining threshold- based PFIs is then described in Section 3.1. The below Table 1 summarizes the symbols used in this paper

\subsection{Mining Probabilistic Frequent Item Sets}

Let $I \subseteq V$ a set of items, or an item set the support of I, denoted by $s(\mathrm{I})$, is the number of transactions in which I appears in a transaction database [4]. In precise databases, $\mathrm{s}(\mathrm{I})$ is a single value. This is no longer true in uncertain databases, because in different possible worlds, $s(I)$ can have different values. Let $S\left(w_{j}, I\right)$ be the support count of $\mathrm{I}$ in possible world $w_{j}$. Then, the probability that $\mathrm{s}(\mathrm{I})$ has a value of $\mathrm{i}$, denoted by $\operatorname{Pr}^{I}(i)$, is

$$
\operatorname{Pr}^{I}(i)=\sum_{w_{j} \in W, S\left(w_{j}, I\right)=i} \operatorname{Pr}\left(w_{j}\right)
$$

Hence, $\operatorname{Pr}^{I}(i)(\mathrm{i}=1, \ldots \ldots, n)$ form a probability mass function (pmf) of $\mathrm{S}(\mathrm{I})$, where $\mathrm{n}$ is the size of database $\mathrm{D}$. Now, let minsupe $(0,1]$ be a percentage value, which is generally used to define minimal support in a deterministic database. An item set $\mathrm{I}$ is said to be frequent in a database $\mathrm{D}$ if $\mathrm{s}(I) \geq \operatorname{msc}(D)$ where $\operatorname{msc}(D)=$ minsup $\times n$ is called the minimal support count of $\mathrm{D}[4]$.

Table 1. Summary of Notations 


\begin{tabular}{|c|c|}
\hline Notation & description \\
\hline $\mathrm{D}$ & An uncertain database of $n$ tuples \\
\hline $\mathrm{V}$ & The set of items that appear in D \\
\hline $\mathrm{v}$ & An item, where $\mathrm{v} \in V$ \\
\hline$t_{j}$ & The $\mathrm{j}$-th tuple in D \\
\hline W & The set of all possible worlds \\
\hline$w_{j}$ & A possible world $\mathrm{w}_{\mathrm{j}} \in W$ \\
\hline I & An itemset, where $\mathrm{I} \subseteq \mathrm{V}$ \\
\hline minsup & A real value between $(0,1]$ \\
\hline $\operatorname{msc}(D)$ & A minimal support count in $\mathrm{D}$ \\
\hline $\mathrm{s}(\mathrm{I})$ & The support count of I in D \\
\hline minprob & A real value between $(0,1]$ \\
\hline $\operatorname{Pr}^{I}(i)$ & $\begin{array}{l}\text { Support prob. ( prob. I has a support count of i } \\
\text { i }\end{array}$ \\
\hline $\operatorname{Pr}_{\text {freq }}(I)$ & Frequentness probability of I \\
\hline$\mu^{I}$ & Expected value of $\mathrm{X}^{\mathrm{I}}$ in $\mathrm{D}$ \\
\hline$d$ & Delta database with $\mathrm{n}^{\mathrm{I}}$ tuples \\
\hline$D^{+}$ & New database with $\mathrm{n}^{+}$tuples \\
\hline$F^{D}$ & Set of all PFIs in D \\
\hline$F_{k}^{D}$ & Set of all K-PFIs in D \\
\hline$C_{k}^{+}$ & Set of size $\mathrm{k}$ candidates for $\mathrm{D}^{+}$ \\
\hline$D B$ & A database, can be $\mathrm{D}, \mathrm{d}$ or $\mathrm{D}^{+}$ \\
\hline$S^{D B}(I)$ & The support count of I in DB \\
\hline $\operatorname{Pr}_{\text {freq }}^{D B}(I)$ & The frequentness probability I in DB \\
\hline$\mu^{I}(D B)$ & The expected value of $X^{I}$ in DB \\
\hline
\end{tabular}

For uncertain databases the frequentness probability of I, denoted by $P r_{\text {freq }}(I)$ is the probability that an item set is frequent [6]. Notice that $\operatorname{Pr}_{\text {freq }}(I)$ can be expressed as

$$
\operatorname{Pr}_{\text {freq }}(I)=\sum_{i \geq m s c(D)} \operatorname{Pr}^{I}(i)
$$

Using frequentness probabilities, the proposed algorithm can determine whether an item set I is frequent. In this paper, the algorithm adopt the definition in [6]: I is a Threshold based PFI if its frequentness probability is larger than some user defined threshold [6]. Formally, given a real value minprob $\epsilon(0,1]$, I is a threshold- based PFI, if

$$
\operatorname{Pr}_{\text {freq }}(I) \geq \text { minprob }
$$

Here the minimum probability (minprob) is the frequentness probability threshold.

\section{EVALUATING S- PMF}

This section deals with the computing method of the probability of the item set which is presented in the dataset. The s-pmf s(I) of item set I plays an important role in determining whether I is a PFI. An interesting observation about $\mathrm{s}(\mathrm{I})$ is that it is essentially the number of successful Poisson trials[29]. To explain, let $X_{j}^{I}$ be a random variable, which is equal to one if $I$ is a subset of the items associated with transaction $t_{j}$ (i.e., I is a subset of $t_{j}$ ) or zero otherwise. Given a database of size $\mathrm{n}$, each $\mathrm{I}$ is associated with random variables $X_{1}^{I}, X_{2}^{I}, \ldots, X_{n}^{I}$. all tuples are independent. Therefore, these $n$ variables are independent, and they represent $n$ Poisson trials. Moreover,

$$
X^{I}=\sum_{j=1}^{n} X_{j}^{I}
$$

follows a Poisson binomial distribution. Next, observe an important relationship between $X^{I}$ and $\operatorname{Pr}^{I}(i)$ (i.e., the probability that the support of I is $i$ )

$$
\operatorname{Pr}^{I}(i)=\operatorname{Pr}\left(X^{I}=i\right)
$$

This is simply because $X^{I}$ is the number of times that I exists in the database. Hence, the $\mathrm{s}^{-}$pmf of I, i.e., $\operatorname{Pr}^{I}(i)$ is the pmf of $X^{I}$, a Poisson binomial distribution. Using (5), it can rewrite (2), which computes the frequentness probability of I, as

$$
\begin{aligned}
\operatorname{Pr}_{\text {freq }}(I) & =\sum_{i \geq m s c(D)} \operatorname{Pr}\left(X^{I}=i\right) \\
& =\operatorname{Pr}\left(X^{I} \geq \operatorname{msc}(D)\right)
\end{aligned}
$$

Therefore, if the cumulative distribution function (cdf) of $X^{I}$ is known, $\left.P r_{\text {freq }}(I) \mathrm{I}\right)$ can also be evaluated. Next, an approach to this cdf, in order to compute $P r_{\text {freq }}(I)$ efficiently is presented.

\subsection{Approximating $\mathrm{s}^{-}$pmf}

From (7), it is possible to express $P r_{\text {freq }}(I)$ as

$$
\operatorname{Pr}_{\text {freq }}(I)=1-\operatorname{Pr}\left(X^{I} \leq m s c(D)-1\right)
$$

For notational convenience, let $P r_{j}^{I}$ be $\operatorname{Pr}\left(I \subseteq t_{j}\right)$.Then, the expected value of $X^{I}$ in $\mathrm{D}$, denoted by $\mu^{I}$, can be computed by

$$
\mu^{I}=\sum_{j=1}^{n} P_{j}^{I}
$$

Since a Poisson binomial distribution can be well approximated by a Poisson distribution [8], (8) can be written as

$$
\operatorname{Pr}_{\text {freq }}(I) \approx 1-F\left(\operatorname{msc}(D)-1, \mu^{I}\right)
$$

Where $\mathrm{F}$ is the cdf of the Poisson distribution with mean $\mu^{\mathrm{I}}$, i.e., $\quad F\left(m s c(D)-1, \mu^{I}\right)=1-\frac{\Gamma\left(m s c(D), \mu^{I}\right)}{(m s c(D)-1) !}$ expressed using the incomplete gamma function

$$
\Gamma(s, x)=\int_{x}^{\infty} t^{s-1} e^{-t} d t
$$

Empirical results may be obtained using this function. To estimate can first compute $\mu^{I}$ by scanning D once and summing up $P_{j}^{I}$ 's for all tuples $t_{j}$ in D.

Then, $F\left(\operatorname{msc}(D)-1, \mu^{I}\right)$ is evaluated, and (10) is used to approximate $P r_{\text {freq }}(I)$. The following theorem can be used to support the proposed algorithm which can be presented as follows

Theorem 1. $P r_{\text {freq }}(I)$, if approximated by (10), increases monotonically with $\mu^{I}$.

Proof. The cdf of a Poisson distribution, $F(i, \mu)$ can be written as 


$$
F(i, \mu)=\frac{\Gamma(i+1, \mu)}{i !}=\frac{\int_{\mu}^{\infty} t^{(i+1)-1} e^{-t} d t}{i !}
$$

Since minsup is fixed and independent of $\mu$, let us examine the partial derivative w.r.t. $\mu$

$$
\begin{aligned}
\frac{\partial F(i, \mu)}{\partial \mu} & =\frac{\partial}{\partial \mu}\left(\frac{\int_{\mu}^{\infty} t^{(i+1)-1} e^{-t} d t}{i !}\right) \\
& =\frac{1}{i !} \frac{\partial}{\partial \mu}\left(\int_{\mu}^{\infty} t^{i} e^{-t} d t\right) \\
& =\frac{1}{i !}\left(-\mu^{i} e^{-\mu}\right) \\
& =-f(i, \mu) \leq 0 .
\end{aligned}
$$

Thus, the cumulative distribution (cdf) of the Poisson distribution $F(i, \mu)$ is monotonically decreasing w.r.t. $\mu$, when $\mathrm{i}$ is fixed. Consequently, $1-F(i-1, \mu)$ increases monotonically with $\mu$. Theorem 1 follows immediately by substituting $i=\operatorname{msc}(D)$.Intuitively, Theorem 1 states that the higher value of $\mu^{I}$, the higher is the chance that $\mathrm{I}$ is a PFI. Next, the fact of how this theorem avoids the costly computations of $\mathrm{F}$, and improves the efficiency of finding threshold-based PFIs was presented.

\section{MINING THRESHOLD BASED PFIs}

It is very difficult to quickly determine whether an item set I is a threshold-based PFI. Since in typical PFI mining algorithm (e.g., [6]), candidate item sets are first generated, before they are tested on whether they are PFI's. In Section 5.1, The method of testing whether I is a threshold-based PFI, without computing its frequentness probability is presented below. The method can be enhanced in Section 5.2. The adaptation of these techniques in an existing PFI- mining algorithm was demonstrated in Section 5.3.

\subsection{PFI Testing}

Given the values of minsup and minprob the algorithm can test whether I is a threshold- based PFI, in three steps.

Step-1. Find a real number $\mu_{\mathrm{m}}$ satisfying the equation:

$$
\text { minprob }=1-F\left(\operatorname{msc}(D)-1, \mu^{I}\right)
$$

The above equation can be solved efficiently by employing numerical methods.

Step-2. Use (9) to compute $\mu^{I}$ Notice that the database D has to be scanned once.

Step-3. If $\mu^{I} \geq \mu_{m}$, The algorithm conclude that I is a PFI, otherwise, I must not be a PFI.

To understand this, first notice that the right side of (11) is the same as that of (10), an expression of frequentness probability. essentially, Step- 1 finds out the value of $\mu_{m}$ that corresponds to the frequentness probability threshold (i.e.,.minprob). In Steps 2 and 3, if $\mu^{\mathrm{I}} \geq \mu_{\mathrm{m}}$, Theorem 2 allows us to deduce that the $P r_{\text {freq }}(I) \geq$ minprob.

Hence, these steps together can test whether an item set is a PFI. In order to verify whether I is a PFI, once $\mu_{\mathrm{m}}$ is found, The algorithm might not have to evaluate $\operatorname{Pr}_{\text {freq }}(I)$. Instead, it compute $\mu^{\mathrm{I}}$ in Step 2, which can be done in $\mathrm{O}(\mathrm{n})$ time. This is a more scalable method compared with solutions in [6] and [35], which evaluate $\operatorname{Pr}_{\text {freq }}(I)$ in $\mathrm{O}\left(\mathrm{n}^{2}\right)$ time. Next, the process of how this method can be further improved is presented in below section .

\subsection{Improving the PFI Testing Process}

In Step 2 of the last section, $\mathrm{D}$ has to be scanned once to obtain $\mu^{\mathrm{I}}$, for every item set I. This can be costly if D is large, and if many item sets need to be tested. For example, in the Apriori algorithm [6], many candidate item sets are generated first before testing whether they are PFIs. next the process of how the PFI testing can still be carried out without scanning the whole database was explained. Let $\mu_{l}^{I}=\sum_{j=1}^{l} p_{j}$, where 1 $\in(0, \mathrm{n}]$. Essentially, $\mu_{l}^{I}$ is the "partial value" of $\mu^{I}$ which is obtained after scanning $l$ tuples. Notice that $\mu_{n}^{I}=\mu^{I}$ Suppose that $\mu_{m}$ has been obtained from (11).

Corollary 1. An item set I cannot be a PFI if there exists $\mathrm{I} \in\left(0, \mu_{\mathrm{m}}\right]$ such that

$$
\mu_{n-i}^{I}<\mu_{m}-i
$$

The above equations can be used to improve the speed of the PFI testing process. Specifically, after a tuple has been scanned, The algorithm checks whether the s-pmf value of the item set exceeds the threshold value; if so, it immediately conclude that I is a PFI. After scanning $n-\mu_{m}$ or more tuples, we examine whether I is not a PFI, by using Corollary 1. These testing procedures continue until the whole database is scanned, yielding $\mu^{\mathrm{I}}$, Then, the alogorithm execute Step 3 (Section 5.1) to test whether I is a PFI.

\section{INCREMENTAL MINING}

Now the method of how to efficiently maintain a set of PFIs in an evolving database is presented here, where new tuples, or transactions, are constantly appended to it. We assume that every tuple has a timestamp attribute, which indicates the time that it is created. This timestamp is not used for mining; it is only used to differentiate new tuples from existing ones. Let $\mathrm{D}$ be the "old" database that contains $\mathrm{n}$ tuples, and $\mathrm{d}$ be a delta database of $n^{\mathrm{I}}$ tuples, whose timestamps are larger than those of tuples in D. Let $\mathrm{D}^{+}$be a "new" database, which is a concatenation of the tuples in $\mathrm{D}$ and $\mathrm{d}$, and has a size of $\mathrm{N}^{+}=$ $\mathrm{n}+\mathrm{n}^{\mathrm{I}}$. Given the set of PFIs and their $\mathrm{s}^{-}$pmfs in $\mathrm{D}$, the goal is to discover PFIs on $\mathrm{D}^{+}$, under the same minsup and minprob values used to mine the PFIs of $D$. The algorithm uses $s^{\mathrm{DB}}(\mathrm{I})$ and $\mathrm{Pr}^{\mathrm{DB}}{ }_{\text {freq }}(\mathrm{I})$ to respectively denote the support count and the frequentness probability of item set $\mathrm{I}$ in some database $\mathrm{DB}$, where $D B$ is in $\left\{D, d, D^{+}\right\}$. The mining problem described above can be treated as a special case of stream mining, which refers to the maintenance of mining results for stream data. Particularly, the database $\mathrm{d}$ as the arrival of $|\mathrm{d}|$ data units from a stream source is assumed. Moreover, assume that the sliding window initially contains $\mathrm{D}$, which then expands to incorporate new stream units. Mining $\mathrm{D}^{+}$is then equivalent to updating the mining results for the arrival of $|\mathrm{d}|$ stream units. In Section 8.3, an adaptation of a stream algorithm in [35] for use in mining is observed. A simple way of obtaining PFIs from $\mathrm{D}^{+}$is to simply rerun a PFI- mining algorithm on it. However, this approach is not very economical, since 1) running a PFI algorithm on a large database is not trivial; and 2) the same algorithm has to be frequently executed if a lot of update activities occur. In fact, if only a few tuples in d are appended to $\mathrm{D}$, it may not be necessary to compute all PFIs on $\mathrm{D}^{+}$from scratch. This is because the PFIs found in $\mathrm{D}^{+}$should not be very different from those discovered in D. Based on this intuition, a mining algorithm that finds PFIs in $\mathrm{D}^{+}$, without rerunning a complete PFI algorithm was designed. This algorithm works the best when the size of $d$ is very small compared with that of $\mathrm{D}$; nevertheless, it works with any size 
of $\mathrm{d}$. The framework of the solution, discovers PFIs in $\mathrm{D}^{+}$, based on the PFIs found in D. this solution is extended to discover PFIs in Section 7.

\subsection{Algorithm}

The algorithm maintains frequent item set results in an evolving database. The proposed algorithm extracts frequent item sets in an "Apriori" fashion: it utilizes a bottom- up approach, where $(\mathrm{k}+1)-$ PFIs are generated from $\mathrm{k}-$ PFIs. The working process of the algorithm is shown in the below diagram.

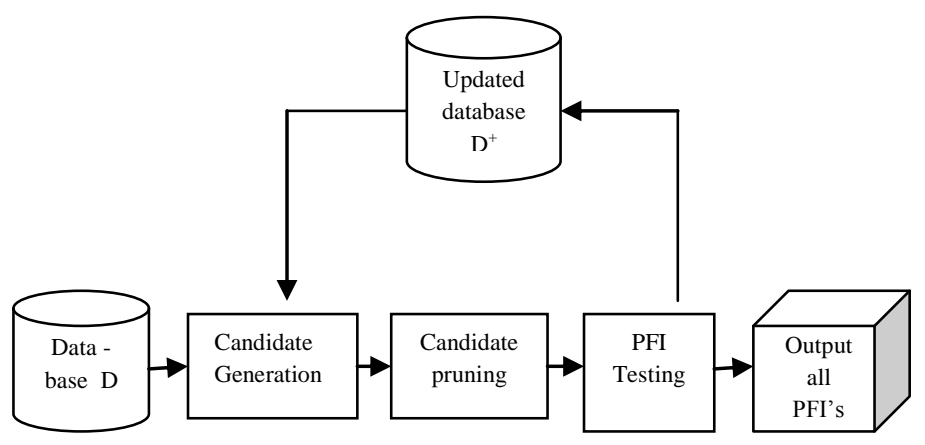

Fig.1: PFI mining from the uncertain database

The algorithm contains three phases. These are given below, 1. Candidate generation. In the first iteration, size -1 item sets that can be 1- PFIs are obtained, using the PFIs discovered from $\mathrm{D}$, as well as the delta database $\mathrm{d}$. In subsequent iterations, this phase produces size $(\mathrm{k}+1)$ candidate item sets, based on the $\mathrm{k}-$ PFIs found in the previous iteration. If no candidates are found, the algorithm halts.

2. Candidate pruning. With the aid of $\mathrm{d}$ and the PFIs found from $\mathrm{D}$, this phase filters the candidate item sets that must not be a PFI.

3. PFI testing. For item sets that cannot be pruned, they are tested to see whether they are the true PFIs. This involves the use of database $\mathrm{D}^{+}$, as well as the $\mathrm{s}^{-}$pmf's of PFIs on $\mathrm{D}$.

Notice that in Phases 1 and 2, only $d$ and the PFIs of D are needed. Since these pieces of information are relatively small in size (compared with $\mathrm{D}$ or $\mathrm{D}^{+}$), they are usually not very expensive to evaluate. Phase 3 involves deriving the s-pmf's of item sets, with the use of $\mathrm{D}^{+}$, and is thus more expensive than other phases. If Phase 2 successfully removes a lot of candidates from consideration, the cost of executing Phase 3 can be reduced.

The above discussion is formalized in the Algorithm-1, which uses the databases D and d, as well as the set of PFIs $\mathrm{F}^{\mathrm{D}}$ collected from $\mathrm{D}$ (e.g., using the method of [6]). The output of the algorithm is a set $\mathrm{F}^{+}$of PFIs for $\mathrm{D}^{+}$, where $F_{k}^{+}=\left\{F_{1}^{+}, F_{2}^{+}, \ldots, F_{n}^{+}\right\}$and $\mathrm{F}_{\mathrm{k}}^{+}$is the set of ' $\mathrm{k}$ - PFIs' for $\mathrm{D}^{+}$ Let $\mathrm{C}_{\mathrm{k}}^{+}$be a set of size- $\mathrm{k}$ candidates found from $\mathrm{D}^{+}$. Initially $\mathrm{k}=1$. The algorithm generates $\mathrm{C}_{1}^{+}$(Phase 1$)$. In the $\mathrm{k}^{\text {th }}$ iteration, first the algorithm removes candidate item sets that cannot be $\mathrm{k}-$ PFIs, from $\mathrm{C}_{\mathrm{k}}^{+}$(Phase 2). if $\mathrm{C}_{\mathrm{k}}^{+}$is not empty, the algorithm performs testing on these candidates, in order to find out the true $\mathrm{k}-$ PFIs (i.e., $\mathrm{F}_{\mathrm{k}}{ }^{+}$,) Phase 3 generates size $(\mathrm{k}+1)$-candidate item sets by using the $\mathrm{k}-$ PFIs. The whole process is repeated until no more candidates are found., The algorithm takes $\mathrm{D}, \mathrm{d}$, minsup, minprob as inputs and produces the output as a set of Probabilistic Frequent item sets (PFIs) in the function $\mathrm{F}$, which is given as follows,

\section{Algorithm- 1 For PFI mining}

Input: Data base D, updated delta database d, minsup, minprob .

Output: PFI's of $\mathrm{D}^{+}, \mathrm{F}_{\mathrm{k}}^{+}=\left\{\mathrm{F}_{1}^{+}, \mathrm{F}_{2}^{+}, \ldots, \mathrm{F}_{\mathrm{n}}^{+}\right\} / / \mathrm{F}_{\mathrm{k}}^{+}$is the set of PFI's.

Method:

if (deltaDb is empty) then

\{

$\mathrm{C}(1)$. Generate( oldDb);

$\mathrm{k}=1$;

while $(\mathrm{C}(\mathrm{k}) !=0)$ do

\{

$\mathrm{C}(\mathrm{k})$.prune(oldDb, F, $\left.\mu_{m}\right)$;

if $(\mathrm{C}(\mathrm{k})$ ! $=0)$ then

$\mathrm{F}=\mathrm{C}(\mathrm{k})$. Test $\left(\right.$ oldDb, d, F, $\left.\mu_{m}\right)$;

else

break;

$\mathrm{C}(\mathrm{k}+1)$.Generate( oldDb );

$\mathrm{k}=\mathrm{k}+1$;

\}

\}

else //deltaDb is not empty

\{

$\mathrm{C}(1)$.Generate(deltaDb);

$\mathrm{k}=1$;

while $(\mathrm{C}(\mathrm{k}) !=0)$

\{

$\mathrm{C}(\mathrm{k})$.prune( deltaDb, F, $\mu_{m}^{I}$ );

if $(\mathrm{C}(\mathrm{k}) !=0)$ then

$\mathrm{F}=\mathrm{C}(\mathrm{k})$.Test(oldDb, d, F, $\left.\mu_{m}^{I}\right)$;

else

break;

$\mathrm{C}(\mathrm{k}+1) \cdot$ Generate $(\mathrm{d})$;

$\mathrm{k}=\mathrm{k}+1$;

\}

return $F_{k}^{+}=\left\{F_{1}^{+}, F_{2}^{+}, \ldots, F_{n}^{+}\right\}$

\section{RESULTS}

Now the experimental results on the data set, called accidents, comes from the Frequent Item set Mining (FIMI) Data Set Repository. This data set is obtained from the National Institute of Statistics (NIS) for the region of Flanders (Belgium), for the period of 1991-2000. The data are obtained from the "Belgian Analysis Form for Traffic Accidents," which are filled out by a police officer for each traffic accident occurring on a public road in Belgium. The data set contains 3,40,184 accident records, with a total of 572 attribute values. On average, each record has 45 attributes. The algorithm uses the first 10k tuples as the default data set as its input. The default value of minsup is 20 percent. To test the mining algorithm, it uses the first 10k tuples as the old database $\mathrm{D}$, and the subsequent tuples as the delta database $\mathrm{d}$. The default size of $d$ is 5 percent of D. , it considers both attribute and tuple uncertainty models. For attribute uncertainty, the existential probability of each attribute is drawn from a Gaussian distribution with mean 0.5 and standard deviation 0.125 . This same distribution is also used to characterize the existential probability of each tuple, for the tuple uncertainty model. The default value of minprob is 0.4 . In the results presented, minsup is shown as a percentage of the data set size $n$. Notice that when the values of minsup or minprob are large, no PFIs can be returned; it do not show the results for these values. The experiments were carried out on the Windows XP operating system, on a machine with a 2.66 
$\mathrm{GHz}$ Intel Core 2 Duo processor and 2 GB memory. The programs were written in Java and compiled with J2SE runtime environment 1.6.0. The proposed algorithm is implemented under the java runtime environment. The algorithm extracts the probabilistic frequent item sets within a fraction of seconds.

\section{DISCUSSION}

Now the comparison of the performance of the PFI mining algorithms mentioned in this paper:1) APM, the Apriori algorithm used in [6] that employs the PFI testing method and 2) PA (Proposed algorithm), the proposed algorithm that uses the improved version of the PFI testing method is discussed here.

Since APM approximates s-pmf by a Poisson distribution, first examine that its accuracy with respect to AP, which yields PFIs based on exact frequentness probabilities. Here, the standard recall and precision measures [7] is used, which quantify the number of negatives and false positives. Specifically, let $F_{A P M}$ be the set of PFIs generated by APM, and $F_{P A}$ be the set of PFIs produced by PA. To compare the existing algorithm with the proposed algorithm, The recall and precision were used. Both recall and precision have values between 0 and 1 . The recall and the precision of APM, relative to $\mathrm{PA}$, are defined as

$$
\begin{aligned}
\text { recall } & =\frac{\left|F_{A P M} \cap F_{A P}\right|}{F_{A P M}} \\
\text { precision } & =\frac{\left|F_{A P M} \cap F_{A P}\right|}{F_{A P}}
\end{aligned}
$$

In these formulas, Also, a higher value reflects a better accuracy. Table 2 shows the recall and the precision of APM (Apriori algorithm), for a wide range of minsup, $\mathrm{n}$, and minprob values. As it can observed that the precision and recall values are always higher than 98 percent. Since The proposed algorithm PA returns the same PFIs as APM, it is also highly accurate result. Both precision and recall are used to compare the performance of mining algorithms.

\section{Table 2. Recall and Precision of APM}

\begin{tabular}{|l|l|l|l|l|l|}
\hline minsup & 0.1 & 0.2 & 0.3 & 0.4 & 0.5 \\
\hline Recall & 1 & 1 & 1 & 1 & 1 \\
\hline Precision & 0.997 & 1 & 1 & 1 & 1 \\
\hline
\end{tabular}

(a) Recall and Precision vs. minsup

\begin{tabular}{|l|l|l|l|l|l|}
\hline minprob & 0.1 & 0.3 & 0.5 & 0.7 & 0.9 \\
\hline Recall & 1 & 1 & 1 & 1 & 1 \\
\hline Precision & 0.986 & 1 & 0.985 & 1 & 1 \\
\hline
\end{tabular}

(b) Recall and Precision vs. minprob

\begin{tabular}{|l|l|l|l|l|l|}
\hline $\mathrm{n}$ & $1 \mathrm{k}$ & $4 \mathrm{k}$ & $10 \mathrm{k}$ & $50 \mathrm{k}$ & $100 \mathrm{k}$ \\
\hline Recall & 1 & 1 & 1 & 1 & 1 \\
\hline Precision & 0.987 & 0.988 & 1 & 1 & 1 \\
\hline
\end{tabular}

(c) Recall and Precision vs. $\mathrm{n}$

\subsection{Efficiency of PA vs. APM}

next compare PA and APM, which both yield PFIs. The given Figure 3(a) shows that PA is faster than APM over different minsup values. minsup is taken on the $\mathrm{X}$-axis and runtime is taken on the Y-axis. On increasing the minsup value the runtime decreases. Fig.3(b) examines the algorithms under a wide range of minprob values. Again, PA runs faster than APM. Fig. 3(b) examines the effect of using different minprob values. Minprob is taken on $\mathrm{X}$-axis and running time is taken on the Y-axis. The details are of these distributions are listed in Table 3.

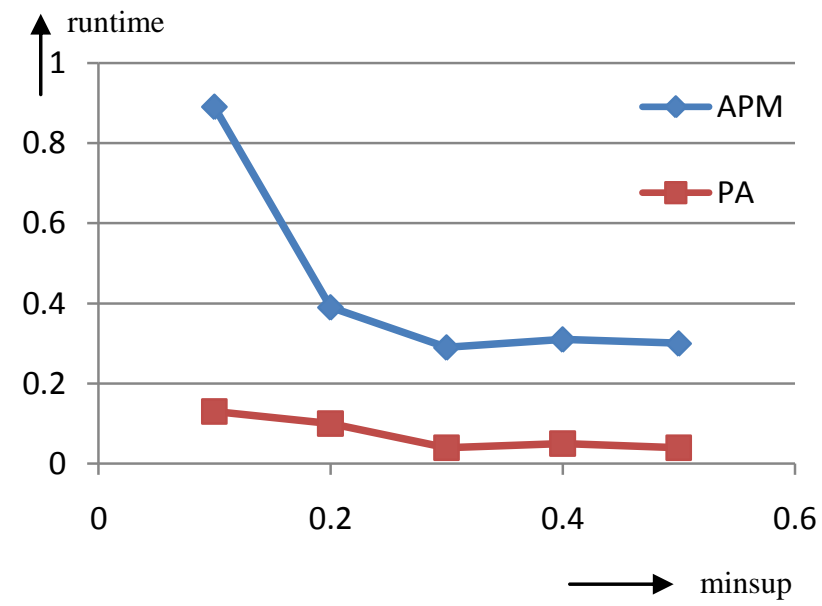

Fig.10(a) minsup vs. runtime

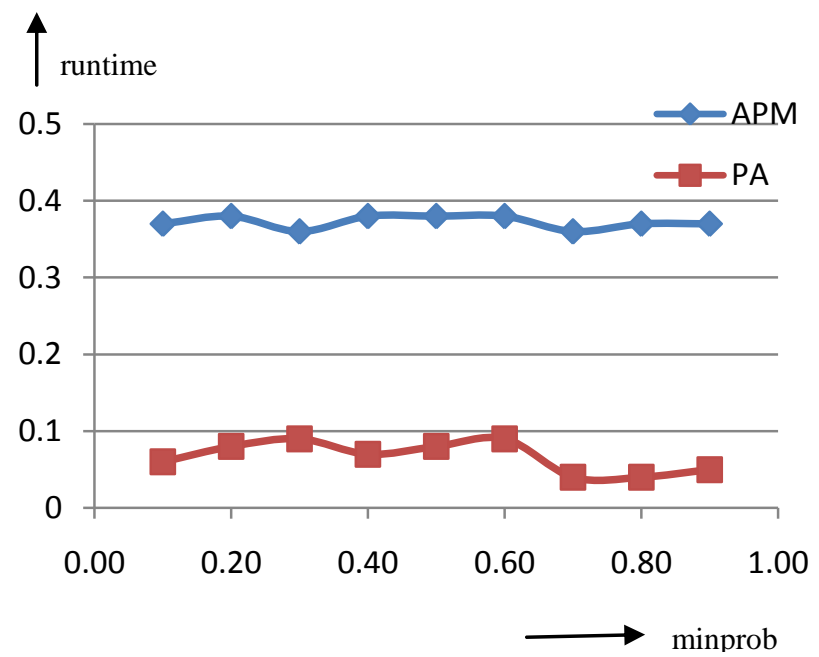

Fig.3(b) minprob vs. runtime

It is observed that the algorithm PA performs better than APM over different types of distributions. The consistently high performance gain demonstrated by AP can be explained. On observing the figure 3(a) the existing algorithm takes longer time than the proposed algorithm(PA), since the on increasing the minsup value against the runtime the curve gradually decreases in existing algorithm and also maintains constant behavior in the proposed algorithm. The graph given in the figure 3(b) depicts the difference in performs between the existing algorithm APM and the proposed algorithm PA. In this graph also the developed or proposed algorithm is faster than the existing algorithm. The below table 3 provides the details of the recall and precision of PA algorithm. 


\section{TABLE 3. Recall and Precision of PA}

\begin{tabular}{|l|l|l|l|l|l|}
\hline minsup & 0.1 & 0.2 & 0.3 & 0.4 & 0.5 \\
\hline Recall & 1 & 1 & 1 & 1 & 1 \\
\hline Precision & 0.998 & 1 & 1 & 1 & 1 \\
\hline
\end{tabular}

(a) Recall and Precision vs. minsup

\begin{tabular}{|l|l|l|l|l|l|}
\hline minprob & 0.1 & 0.3 & 0.5 & 0.7 & 0.9 \\
\hline Recall & 1 & 1 & 1 & 0.970 & 1 \\
\hline Precision & 0.986 & 1 & 1 & 1 & 1 \\
\hline
\end{tabular}

(b) Recall and Precision vs. minprob

\begin{tabular}{|l|l|l|l|l|l|}
\hline $\mathrm{n}$ & $1 \mathrm{k}$ & $5 \mathrm{k}$ & $10 \mathrm{k}$ & $50 \mathrm{k}$ & $100 \mathrm{k}$ \\
\hline Recall & 1 & 1 & 1 & 1 & 1 \\
\hline Precision & 1 & 1 & 1 & 1 & 0.985 \\
\hline
\end{tabular}

(c) Recall and Precision vs. $n$

The experiments on the tuple uncertainty model and on the synthetic data set were also performed. Since they are similar to the results presented above, The most representative ones. For the accuracy aspect, the recall and precision values of approximate results on these data sets are still higher than 98 percent. Thus, the proposed algorithm can return accurate results.

\section{CONCLUSIONS}

In this paper, we propose a approach to extract thresholdbased PFIs from large uncertain databases. Its main idea is to the s-pmf of a PFI by some common probability model, so that a PFI can be verified quickly. This approach supports retrieving PFIs from evolving databases. The experimental results show that the proposed algorithm is highly efficient and accurate. It supports both attribute- and tuple uncertain data approach to develop other mining algorithm (e.g., clustering and classification) on uncertain data. It is also interesting to study efficient mining algorithm for handling tuple. Another interesting work is to investigate PFI mining algorithm for probability models that capture correlation among attributes and tuples.

\section{REFERENCES}

[1] A. Veloso, W. Meira Jr., M. de Carvalho, B. Po^ssas, S. Parthasarathy, and M.J. Zaki, "Mining Frequent Itemsets in Evolving Databases," Proc. Second SIAM Int'l Conf. Data Mining (SDM), 2002.

[2] C. Aggarwal, Y. Li, J. Wang, and J. Wang, "Frequent Pattern Mining with Uncertain Data, ", Proc. 15th ACM SIGKDD Int'l Conf. Knowledge Discovery and Data Mining (KDD), 2009.

[3] C. Aggarwal and P. Yu, "A Survey of Uncertain Data Algorithm and Applications," IEEE Trans Knowledge and Data Eng., vol. 21, no. 5, pp. 609-623, May 2009.[4] R. Agrawal, T. Imielipnski, and A. Swami, "Mining Association Rules between Sets of Items in Large Databases," Proc.ACM SIGMOD Int'l Conf. Management of Data, 1993.

[5 ] O. Benjelloun, A.D. Sarma, A. Halevy, and J. Widom, "ULDBs: Databases with Uncertainty and Lineage," Proc. 32ndInt'l Conf. Very Large Data Bases (VLDB), 2006.

[6] T. Bernecker, H. Kriegel, M. Renz, F. Verhein, and A. Zuefle,"Probabilistic Frequent Itemset Mining in Uncertain Databases," Proc. 15th ACM SIGKDD Int'l
Conf. Knowledge Discovery and Data Mining (KDD), 2009.

[7] C.J. van Rijsbergen, Information Retrieval. Butterworth, 1979.

[8] L.L. Cam, "An Approximation Theorem for the Poisson Binomial Distribution," Pacific J. Math., vol. 10, pp. 1181- 1197,1960.

[9] H. Cheng, P. Yu, and J. Han, “ Frequent Itemset Mining in the Presence of Random Noise," Proc. Soft Computing for Knowledge Discovery and Data Mining, pp. 363-389, 2008.

[10] R. Cheng, D. Kalashnikov, and S. Prabhakar, "Evaluating Probabilistic Queries over Imprecise Data," Proc. ACM SIGMOD Int'l Conf. Management of Data, 2003.

[11] D. Cheung, J. Han, V. Ng, and C. Wong, "Maintenance of Discovered Association Rules in Large Databases: An Updating Technique," Proc. 12th Int'l Conf. Data Eng. (ICDE), 1996.

[12] D. Cheung, S.D. Lee, and B. Kao, “A General Technique for Maintaining Discovered Association Rules," Proc. fifth Int'1 Conf. Database Systems for Advanced Applications (DASFAA), 1997.

[13] W. Cheung and O.R. Zaı"ane, " Mining of Frequent Patterns without Candidate Generation or Support Constraint," Proc. Seventh Int'l Database Eng. and Applications Symp. (IDEAS), 2003.

[14] C.K. Chui, B. Kao, and E. Hung, "Mining Frequent Itemsets from Uncertain Data," Proc. 11th Pacific-Asia Conf. advancesin Knowledge Discovery and Data Mining (PAKDD), 2007.

[15] G. Cormode and M. Garofalakis, "Sketching Probabilistic Data Streams," Proc. ACM SIGMOD Int'l Conf. Management of Data, 2007.

[16] N. Dalvi and D. Suciu, "Efficient Query Evaluation on Probabilistic Databases," Proc. 13th Int'l Conf. Very Large Data Bases (VLDB), 2004.

[17] A. Deshpande, C. Guestrin, S.Madden, J. Hellerstein, and W. Hong, "Model-Driven Data Acquisition in Sensor Networks," Proc. 13th Int'l Conf. Very Large Data Bases (VLDB), 2004.

[18] J. Han, J. Pei, and Y. Yin,"Mining Frequent Patterns without Candidate Generation," Proc. ACM SIGMOD Int'l Conf. Management of Data, 2000.

[19] J. Huang, "MayBMS: A Probabilistic Database Management System," Proc. 35th ACM SIGMOD Int'l Conf. Management of Data, 2009.

[20] R. Jampani, L. Perez, M. Wu, F. Xu, C. Jermaine, and P. Haas, "MCDB: A Monte Carlo Approach to Managing Uncertain Data," Proc. ACM SIGMOD Int'l Conf. Management of Data 2008

[21] J. Ren, S.D. Lee, X. Chen, B. Kao, R. Cheng, and D.W. Cheung" Naive Bayes Classification of Uncertain Data," Proc. IEEE Ninth Int'l Conf. Data Mining (ICDM), 2009.

[22] N. Khoussainova, M. Balazinska, and D. Suciu, "Towards Correcting Input Data Errors Probabilistically Using Integrity Constraints," Proc. Fifth ACM Int'l Workshop Data Eng. For Wireless and Mobile Access (MobiDE), 2006.

[23] H. Kriegel and M. Pfeifle, "Density-Based Clustering of Uncertain Data," Proc. ACM SIGKDD Int'l Conf. Knowledge Discovery in Data Mining (KDD), 2005.[24] C. Kuok, A. Fu, and M. Wong, "Mining Fuzzy Association Rules in Databases," SIGMOD Record, vol. 27, no. 1, pp. 41-46, 1998. Proc. IEEE Fifth Int'l Conf. Data Mining (ICDM), 2005 
[25] C.K.-S. Leung, Q.I. Khan, and T. Hoque, "Cantree: A Tree Structure for EfficientMining of Frequent Patterns,". Mining (ICDM), 2005.

[26] A.Lu, Y. Ke, J. Cheng, and W. Ng, "Mining Vague Association Rules," Proc. 12th Int'l Conf. Database Systems for Advanced Applications (DASFAA), 2007.[27] M. Mutsuzaki, "Trio-One: Layering Uncertainty and

Lineage on a Conventional DBMS," Proc. Third Biennial Conf. Innovative Data Systems Research (CIDR), 2007.[28] P. Sistla, O. Wolfson, S. Chamberlain, and S. Dao, "Querying the Uncertain Position of Moving Objects," Temporal Databases: Research and Practice, Springer Verlag, 1998.

[29] C. Stein, Computation of Expectations, Lecture Notes Monograph Series, vol. 7, Inst. of Math. Statistics, 1986.

[30] L. Sun, R. Cheng, D.W. Cheung, and J. Cheng, "Mining Uncertain Data with Probabilistic Guarantees," Proc. $16^{\text {th }}$ ACM SIGKDD Int'l Conf. Knowledge Discovery and Data Mining, 2010.

[31] T. Jayram et al., "Avatar Information Extraction System,” IEEE Data Eng. Bull., vol. 29, no. 1, pp. 40-48, Mar. 2006.

[32] S. Tsang, B. Kao, K.Y. Yip, W.-S. Ho, and S.D. Lee., "Decision Trees for Uncertain Data," Proc. IEEE Int'l Conf. Data Eng. (ICDE), 2009.

[33] L. Wang, R. Cheng, S.D. Lee, and D. Cheung, "Accelerating Probabilistic Frequent Itemset Mining: A Approach," Proc. 19 ${ }^{\text {th }}$ ACM Int'l Conf. Information and Knowledge Management (CIKM), 2010.

[34] M. Yiu, N. Mamoulis, X. Dai, Y. Tao, and M. Vaitis, "Efficient Evaluation of Probabilistic Advanced Spatial Queries on existentially Uncertain Data," IEEE Trans Knowledge and Data Eng., vol. 21, no. 9, pp. 108-122, Jan. 2009.

[35] Q. Zhang, F. Li, and K. Yi, "Finding Frequent Items in Probabilistic Data," Proc. ACM SIGMOD Int'l Conf. Management of Data, 2008. 